\title{
A note on $\delta$-Jordan homomorphism on Banach algebras
}

\author{
A. Zivari-Kazempour \\ Department of Mathematics, Ayatollah Borujerdi University, Borujerd, Iran \\ Email: zivari@abru.ac.ir
}

Copyright (C2014 A. Zivari-Kazempour. This is an open access article distributed under the Creative Commons Attribution License, which permits unrestricted use, distribution, and reproduction in any medium, provided the original work is properly cited.

\begin{abstract}
In this paper we show that, under special hypotheses, each $\delta$-Jordan homomorphism $\varphi$ between Banach algebras $\mathcal{A}$ and $\mathcal{B}$ is continuous and almost multiplicative.
\end{abstract}

Keywords: $\delta$-Jordan homomorphism, almost multiplicative, Semisimple.

\section{Introduction}

Let $\mathcal{A}$ and $\mathcal{B}$ be Banach algebras and $\varphi: \mathcal{A} \longrightarrow \mathcal{B}$ be a linear map. Then $\varphi$ is called Jordan homomorphism if $\varphi\left(a^{2}\right)=\varphi(a)^{2}$ for all $a \in \mathcal{A}[4]$, and it is called $\delta$-Jordan homomorphism if there exist $\delta>0$ such that

$$
\left\|\varphi\left(a^{2}\right)-\varphi(a)^{2}\right\| \leq \delta\|a\|^{2}, \quad(a \in \mathcal{A})
$$

Moreover, $\varphi$ is said to be multiplicative, if $\varphi(a b)=\varphi(a) \varphi(b)$ for all $a, b \in \mathcal{A}$, and it is said to be almost multiplicative [3], if there exist $\xi>0$ such that

$$
\|\varphi(a b)-\varphi(a) \varphi(b)\| \leq \xi\|a\|\|b\|, \quad(a, b \in \mathcal{A})
$$

It is obvious that if $\varphi$ is multiplicative (almost multiplicative), then it is Jordan homomorphism $(\delta$-Jordan homomorphism), but the converse is false, in general.

In [5], Zelazko show that if $\mathcal{B}$ is commutative and semisimple, then each Jordan homomorphism $\varphi: \mathcal{A} \longrightarrow \mathcal{B}$ is multiplicative. See also [6] for another characterization of this result.

In this paper we investigate a similar result for $\delta$-Jordan homomorphism and then we give a sufficient conditions that each $\delta$-Jordan homomorphism $\varphi: \mathcal{A} \longrightarrow \mathcal{B}$ to be almost multiplicative (Theorem 2.5 below).

It is well-known that every multiplicative linear functional $\varphi$ on Banach algebra $\mathcal{A}$ is continuous and $\|\varphi\| \leq 1$, see [2] for example.

In [3], Jarosz generalized this result and proved the following Theorem.

Theorem 1.1 Let $\varphi: \mathcal{A} \longrightarrow \mathbb{C}$ be an almost multiplicative linear functional, then $\varphi$ is continuous and \|\|$\leq 1+\xi$ 
The next result, which is a generalization of Jarosz's theorem, obtained in [1].

Theorem 1.2 Let $\varphi: \mathcal{A} \longrightarrow \mathcal{B}$ be an almost multiplicative linear map. If $\mathcal{B}$ is semisimple, then $\varphi$ is continuous and $\|\varphi\| \leq 1+\xi$.

\section{2. $\delta$-Jordan homomorphism}

The next result show that Theorem 1.1 is valid for $\delta$-Jordan homomorphism instead of almost multiplicative.

Theorem 2.1 Let $\varphi$ be a $\delta$-Jordan homomorphism from Banach algebra $\mathcal{A}$ into $\mathbb{C}$. Then $\varphi$ is continuous and $\|\varphi\| \leq 1+\delta$.

Proof. By definition we have $\|\varphi\|=\sup \{|\varphi(a)|: a \in \mathcal{A},\|a\|=1\}$, thus for $0<\lambda<\sqrt{\delta}$, there exist $a \in \mathcal{A}$ with $\|a\|=1$ and $\|\varphi\|-\lambda<|\varphi(a)|$. Then

$$
|\varphi(a)|^{2}-\left|\varphi\left(a^{2}\right)\right|=\left|\varphi(a)^{2}\right|-\left|\varphi\left(a^{2}\right)\right| \leq\left|\varphi\left(a^{2}\right)-\varphi(a)^{2}\right| \leq \delta,
$$

therefore

$$
|\varphi(a)|^{2} \leq\left|\varphi\left(a^{2}\right)\right|+\delta
$$

Since $\|a\|=1$, we have

$$
(\|\varphi\|-\lambda)^{2}<|\varphi(a)|^{2} \leq\left|\varphi\left(a^{2}\right)\right|+\delta \leq\|\varphi\|+\delta .
$$

Letting $\lambda \longrightarrow 0$, so $\|\varphi\|^{2}-\|\varphi\| \leq \delta$. Then

$$
(2\|\varphi\|-1)^{2} \leq 1+4 \delta
$$

It follows that

$$
\|\varphi\| \leq \frac{1+\sqrt{1+4 \delta}}{2} \leq 1+\delta .
$$

Corollary 2.2 Let $\varphi: \mathcal{A} \longrightarrow \mathbb{C}$ be a $\delta$-Jordan homomorphism. Then for all $\lambda \in \mathbb{C},(1+\lambda) \varphi$ is $\delta$-Jordan homomorphism.

Theorem 2.3 Let $\mathcal{A}$ and $\mathcal{B}$ be two Banach algebras and $\varphi: \mathcal{A} \longrightarrow \mathcal{B}$ be a $\delta$-Jordan homomorphism. If $\mathcal{B}$ is semisimple, then $\varphi$ is continuous.

Proof. Let $\psi: \mathcal{B} \longrightarrow \mathbb{C}$ be a $\delta$-Jordan homomorphism. Then $\psi$ is bounded by Theorem 2.1 , so we have

$$
\left|\psi \circ \varphi\left(a^{2}\right)-(\psi \circ \varphi(a))^{2}\right| \leq\|\psi\|\left\|\varphi\left(a^{2}\right)-\varphi(a)^{2}\right\| \leq(1+\delta) \delta\|a\|^{2} .
$$

Therefore $\psi \circ \varphi$ is a $\eta$-Jordan homomorphism, where $\eta=\delta(1+\delta)$, thus it is continuous by above Theorem. Suppose that $\left(a_{n}\right)$ be a sequence in $\mathcal{A}$ such that $\lim _{n} a_{n}=a$ and $\lim _{n} \varphi\left(a_{n}\right)=b$. Then

$$
\psi(b)=\psi\left(\lim _{n} \varphi\left(a_{n}\right)\right)=\lim _{n} \psi \circ \varphi\left(a_{n}\right)=\psi \circ \varphi(a)
$$

thus, $\psi(b-\varphi(a))=0$. Since $\mathcal{B}$ is semisimple, we get $\varphi(a)=b$. Therefore $\varphi$ is continuous by the close graph Theorem.

The norm $\|$.$\| on a Banach algebra \mathcal{A}$ is called uniform, if $\left\|a^{2}\right\|=\|a\|^{2}$ for all $a \in \mathcal{A}$. The uniform Banach algebra is a Banach algebra with uniform norm.

The proof of the next result is same as of Theorem 2.1.

Theorem 2.4 Let $\varphi$ be a $\delta$-Jordan homomorphism from Banach algebra $\mathcal{A}$ into a uniform Banach algebra $\mathcal{B}$. Then $\|\varphi\| \leq 1+\delta$.

The following theorem, which is the main one in the paper, is criterion for a $\delta$-Jordan homomorphism to be almost multiplicative.

Theorem 2.5 Let $\mathcal{A}$ and $\mathcal{B}$ be two commutative Banach algebras and $\varphi: \mathcal{A} \longrightarrow \mathcal{B}$ be a $\delta$-Jordan homomorphism. Then $\varphi$ is almost multiplicative. 
Proof. Let $a, b \in \mathcal{A}$ and $\|a\|=\|b\|=1$. Since $\mathcal{A}$ and $\mathcal{B}$ are commutative, we get

$$
\varphi(a b)-\varphi(a) \varphi(b)=\frac{1}{4}\left[\varphi\left(u^{2}\right)-\varphi(u)^{2}-\varphi\left(v^{2}\right)+\varphi(v)^{2}\right],
$$

where $u=a+b$ and $v=a-b$. Hence

$$
\|\varphi(a b)-\varphi(a) \varphi(b)\| \leq \frac{1}{4}\left\|\varphi\left(u^{2}\right)-\varphi(u)^{2}\right\|+\frac{1}{4}\left\|\varphi\left(v^{2}\right)-\varphi(v)^{2}\right\| \leq \frac{\delta}{4}\left(\|u\|^{2}+\|v\|^{2}\right) \leq 2 \delta .
$$

Therefore,

$$
\|\varphi(a b)-\varphi(a) \varphi(b)\| \leq 2 \delta\|a\|\|b\| .
$$

Put $\xi=2 \delta$, then for all $a, b \in \mathcal{A}$, with $\|a\|=\|b\|=1$, we have

$$
\|\varphi(a b)-\varphi(a) \varphi(b)\| \leq \xi\|a\|\|b\| .
$$

Now suppose that $a, b \in \mathcal{A}$ be arbitrary. Take $x=a /\|a\|$ and $y=b /\|b\|$. Then $\|x\|=\|y\|=1$, so by above argument we get

$$
\|\varphi(x y)-\varphi(x) \varphi(y)\| \leq \xi\|x\|\|y\| .
$$

Since $\|x\|=\|y\|=1$, we deduce

$$
\|\varphi(a b)-\varphi(a) \varphi(b)\| \leq \xi\|a\|\|b\|,
$$

for all $a, b \in \mathcal{A}$. This complete the proof.

Theorem 2.6 Let $\varphi$ be a almost multiplicative linear functional on Banach algebra $\mathcal{A}$, and $\psi \in \mathcal{A}^{\prime}$. If for all $a \in \mathcal{A}$,

$$
|\varphi(a)-\psi(a)|<\varepsilon,
$$

then $\psi$ is almost multiplicative.

Proof. Suppose that $a, b \in \mathcal{A}$ and $\|a\|=\|b\|=1$. Then

$$
\begin{aligned}
|\varphi(a) \varphi(b)-\psi(a) \psi(b)| & \leq|\varphi(b)||\varphi(a)-\psi(a)|+|\varphi(a)-\psi(a)||\varphi(b)-\psi(b)|+|\varphi(a)||\varphi(b)-\psi(b)| \\
& \leq \varepsilon(|\varphi(a)|+|\varphi(b)|)+\varepsilon^{2} \\
& \leq 2 \varepsilon\|\varphi\|+\varepsilon^{2} \\
& \leq 2 \varepsilon(1+\xi)+\varepsilon^{2} .
\end{aligned}
$$

Take $\eta=2 \varepsilon(1+\xi)+\varepsilon^{2}$, then

$$
|\psi(a b)-\psi(a) \psi(b)| \leq|\psi(a b)-\varphi(a b)|+|\varphi(a b)-\varphi(a) \varphi(b)|+|\varphi(a) \varphi(b)-\psi(a) \psi(b)| \leq \varepsilon+\xi+\eta .
$$

Hence, for all $a, b \in \mathcal{A}$ with $\|a\|=\|b\|=1$, we have

$$
|\psi(a b)-\psi(a) \psi(b)| \leq(\varepsilon+\xi+\eta)\|a\|\|b\| .
$$

Thus $\psi$ is almost multiplicative. The result follows for arbitrary non-zero elements $a, b \in \mathcal{A}$, if we replacing $a$ by $a /\|a\|$ and $b$ by $b /\|b\|$ in above inequity.

\section{References}

[1] E. Ansari-Piri and N. Eghbali, "A note on multiplicative and almost multiplicative linear maps", Honam Math. J, Vol. 4, (2005), pp. 641-647.

[2] F. F. Bonsall and J. Duncan, Complete normed algebra, Springer-Verlag, New York, (1973).

[3] K. Jarosz, "Perturbation of Banach algebras", Lecture Notes in Mathematics, Springer-verlag, (1985).

[4] T. Palmer, Banach algebras and the general theory $C^{*}$-algebras, Vol I. Cambridge: Univ Press, (1994).

[5] W. Zelazko, "A characterization of multiplicative linear functionals in complex Banach algebras", Studia Math, Vol. 30 (1968), pp. 83-85.

[6] A. Zivari-kazempour, "A characterization of Jordan homomorphism on Banach algebras", Chinese J. Math, Vol. 2014, (2014), 3 pages. 